\title{
Erratum to: Advances in Artificial Intelligence
}

\author{
Richard Khoury $^{1(\mathbb{})}$ and Christopher Drummond ${ }^{2}$ \\ ${ }^{1}$ Lakehead University, Thunder Bay ON, Canada \\ richard.khoury@lakeheadu.ca \\ 2 National Research Council Canada, Ottawa, Canada \\ christopher.drummond@nrc-cnrc.gc.ca
}

\section{Erratum to: \\ R. Khoury and C. Drummond (Eds.) Advances in Artificial Intelligence DOI: 10.1007/978-3-319-34111-8}

The affiliation in front matter Dr. John Oommen was not complete. The complete information is given below:

The author is also an Adjunct Professor with the University of Agder in Grimstad, Norway.

\section{Erratum to:}

\section{Chapter 7: R. Khoury and C. Drummond (Eds.) Advances in Artificial Intelligence DOI: 10.1007/978-3-319-34111-8_7}

In the original version, the references [4], [8], [14], and [15] were wrong. It should be read as follows:

1) Reference 4: It should be "De Melo, C.M., Carnevale, P., Read, S., Antos, D., Gratch, J.: Bayesian model of the social effects of emotion in decision-making in multiagent systems. In: Proceedings of AAMAS, vol. 1, pp. 55-62" instead of "De Melo, C.M., Carnevale, P., Read, S., Antos, D., Gratch, J.: Bayesian model of the social effects of emotion in decision-making in multiagent systems. Science 1, 55-62 (2012)"

2) Reference 8: It should be "Hoey, J., Schröder, T., Alhothali, A.: Affect control processes: Intelligent affective interaction using a partially observable Markov decision process. Artif. Intell. 230, 134-172 (2016)" instead of "Hoey, J., Schröder, T., Alhothali, A.: Affect control processes: intelligent affective interaction using a partially observable Markov decision process. Science 230, 134-172 (2016)"

\footnotetext{
The updated original online version for this Book can be found at 10.1007/978-3-319-34111-8 The updated original online version for this Chapter can be found at 10.1007/978-3-319-34111-8_7 
3) Reference 14: It should be "Sequeira, P., Melo, F.S., Paiva, A.: Learning by appraising: an emotion-based approach to intrinsic reward design. Adapt. Behav. 22(5), 330-349 (2014)" instead of "Sequeira, P., Melo, F.S., Paiva, A.: Learning by appraising: an emotion-based approach to intrinsic reward design. Science 22(5), 330-349 (2014)"

4) Reference 15: It should be "Squazzoni, F., Jager, W., Edmonds, B.: Social simulation in the social sciences a brief overview. Soc. Sci. Comput. Rev. 32(3), 279-294 (2014)" instead of "Squazzoni, F., Jager, W., Edmonds, B.: Social simulation in the social sciences a brief overview. Science 32(3), 279-294 (2014)" 